\title{
Left Bundle Branch Area Pacing guided by Continuous Uninterrupted Monitoring of the unipolar Pacing Characteristics.
}

\author{
Kris Gillis ${ }^{1}$, Jean-Yves Wielandts ${ }^{2}$, Gabriela Hilfiker ${ }^{1}$, Louisa O’Neill ${ }^{1}$, Alina Vlase ${ }^{3}$, \\ Sebastien Knecht ${ }^{1}$, Mattias Duytschaever ${ }^{1}$, Rene Tavernier $^{1}$, and Jean-Benoit le Polain de \\ Waroux ${ }^{1}$
}

${ }^{1}$ AZ Sint-Jan Brugge-Oostende AV

${ }^{2}$ AZ Sint-Jan AV

${ }^{3} \mathrm{CHwapi}$

July 19, 2021

\begin{abstract}
Introduction. During left bundle branch area pacing (LBBAP) lead implantation, intermittent monitoring of unipolar pacing characteristics validates LBB capture and can detect septal perforation. We aimed to demonstrate that continuous uninterrupted unipolar pacing from an inserted lead stylet (LS) is feasible and facilitates LBBAP implantation. Methods. Thirty patients (mean age $76 \pm 14$ years) were implanted with stylet-driven pacing lead (Biotronik Solia S60). In 10 patients (validation-group) conventional, interrupted implantation was performed, with comparison of unipolar pacing characteristics between LS and connector-pin (CP)-pacing after each rotation step. In 20 patients (feasibility-group) performance and safety of uninterrupted implantation during continuous pacing from the LS were analyzed. Results. In the validation-group, LS and CP-pacing impedances were highly correlated $\left(\mathrm{R}^{2}=0.95, \mathrm{p}<0.0001\right.$, bias $\left.12 \pm 37\right)$. Pacing characteristics from LS and CP showed comparable sensed electrograms and paced QRS morphologies. In the feasibility-group, continuous LS-pacing allowed beat-to-beat monitoring of impedance and QRS morphology to guide implantation. This resulted in successful LBBAP in all patients, after a mean of $1+-0$ attempts, with mean threshold $0.81+-0.4 \mathrm{~V}$, median sensing $6.5 \mathrm{mV}$ [IQR 4.4-9.5] and mean impedance $624+-$ 101, and positive LBBAP-criteria with median paced QRS duration 120ms [IQR 112-152ms] and median pLVAT 73ms [IQR 68-80.5ms]. No septal perforation occurred. Conclusion. Unipolar pacing from the LS allows accurate determination of pacing impedance and generates similar paced QRS morphologies and equal sensed electrograms, compared to CP pacing. Continuous LS pacing allows real-time monitoring of impedance and paced QRS morphology, which facilitates a safe and successful LBBAP lead implantation.
\end{abstract}

\section{Left Bundle Branch Area Pacing guided by Continuous Uninterrupted Monitoring of the Unipolar Pacing Characteristics}

Kris Gillis, MD, PhD*; Jean-Yves Wielandts, MD, MSc, PhD*; Gabriela Hilfiker MD*; Louisa O'Neill, MD, $\mathrm{PhD}^{*}$; Alina Vlase, MD; Sebastien Knecht, $\mathrm{MD}, \mathrm{PhD}^{*}$; Mattias Duytschaever, $\mathrm{MD}, \mathrm{PhD}^{*} \#$; Rene Tavernier, $\mathrm{MD}, \mathrm{PhD}^{*}$ and Jean-Benoit le Polain de Waroux MD, PhD*

Affiliations:* Department of Cardiology, Sint-Jan Hospital, Bruges, Belgium

\# Department of Internal Medicine, Ghent University, Belgium

\section{Corresponding author:}

Kris Gillis

Department of Cardiology, AZ Sint-Jan Brugge Oostende AV, 8000 Bruges, Belgium 
E-mail: gilliskris@yahoo.com

Tel: +325045 2670; Fax: +32 50452679

Short title: Impedance and QRS guided LBBAP

Keywords: His bundle pacing, Left bundle branch area pacing, Physiologic pacing,

Disclosures: Pr. Jean-Benoit le Polain de Waroux reports non-significant speaker fees and honoraria for proctoring and teaching activities from Medtronic, Boston Scientific, Abbott and Biotronik.

\section{Funding: None}

\section{ABSTRACT}

Introduction . During left bundle branch area pacing (LBBAP) lead implantation, intermittent monitoring of unipolar pacing characteristics validates LBB capture and can detect septal perforation. We aimed to demonstrate that continuous uninterrupted unipolar pacing from an inserted lead stylet (LS) is feasible and facilitates LBBAP implantation.

Methods. Thirty patients (mean age $76 \pm 14$ years) were implanted with stylet-driven pacing lead (Biotronik Solia S60). In 10 patients (validation-group) conventional, interrupted implantation was performed, with comparison of unipolar pacing characteristics between LS and connector-pin (CP)-pacing after each rotation step. In 20 patients (feasibility-group) performance and safety of uninterrupted implantation during continuous pacing from the LS were analyzed.

Results. In the validation-group, LS and CP-pacing impedances were highly correlated $\left(\mathrm{R}^{2}=0.95, \mathrm{p}<0.0001\right.$, bias $12 \pm 37 \Omega)$. Pacing characteristics from LS and CP showed comparable sensed electrograms and paced QRS morphologies. In the feasibility-group, continuous LS-pacing allowed beat-to-beat monitoring of impedance and QRS morphology to guide implantation. This resulted in successful LBBAP in all patients, after a mean of $1 \pm 0$ attempts, with mean threshold $0.81 \pm 0.4 \mathrm{~V}$, median sensing $6.5 \mathrm{mV}$ [IQR 4.4-9.5] and mean impedance $624 \pm 101 \Omega$, and positive LBBAP-criteria with median paced QRS duration 120ms [IQR 112-152ms] and median pLVAT 73ms [IQR 68-80.5ms]. No septal perforation occurred.

Conclusion. Unipolar pacing from the LS allows accurate determination of pacing impedance and generates similar paced QRS morphologies and equal sensed electrograms, compared to CP pacing. Continuous LS pacing allows real-time monitoring of impedance and paced QRS morphology, which facilitates a safe and successful LBBAP lead implantation.

\section{Keywords}

His bundle pacing, Left bundle branch area pacing, Physiologic pacing.

\section{Introduction}

Left bundle branch (LBB) area pacing (LBBAP) has been recently introduced as an attractive alternative to His Bundle Pacing (HBP) to deliver physiological pacing.[1-6] Compared to HBP, LBBAP benefits of lower pacing thresholds, higher $\mathrm{R}$ wave amplitude, less atrial far-field oversensing and more stable lead position. To engage the left septal conduction system and obtain physiologic depolarization of the left ventricle (LV), deep implantation of the LBBAP lead in the interventricular septum is essential. As a result, the implant procedure is associated with a significant risk of perforation into the LV cavity around 3\%.[3,4] To prevent perforation, recent published LBBAP experience suggests controlling the penetration depth of the lead repeatedly (using sheath angiography), and to monitor the unipolar pacing impedance, the paced QRS morphology and unipolar EGMs (for LBB potential). However, this requires repetitive interruption of the implantation, to connect the programmer to the connector pin $(\mathrm{CP})$ of the lead. As initial transseptal deployment of the lead is based on the assumption of the septal wall thickness, first evaluation rarely confirms optimal lead positioning. Consequently, if no LV perforation has occurred, careful further advancement of the lead is needed until criteria of LBB capture are obtained. Recently, LBBAP using stylet-driven (SD) 
leads has demonstrated similar high implant success rate when compared to the mostly used lumen-less Medtronic Select Secure lead.[7] Interestingly, the use of SD leads offers the unique opportunity to perform unipolar pacing on the inserted lead stylet (LS) instead of the CP, with the advantage that continuous unipolar pacing is possible during lead rotations for implantation.

In the present study, we aimed first to validate that unipolar pacing from LS and CP results in similar pacing characteristics. Secondly, we aimed to demonstrate that uninterrupted lead implantation guided by continuous unipolar pacing and monitoring of the impedance, QRS morphology, and peak left ventricular activation time (pLVAT) is a safe and efficient technique to obtain LBBAP.

\section{Methods}

\subsection{General methodology}

Between December 2020 and March 2021, 30 consecutive patients with bradycardia or heart failure indications were implanted with a LBBAP at the Heart Centre AZ Sint-Jan, Bruges, Belgium. All procedures were performed using a 5.6 Fr. stylet-driven pacing lead with an extendable helix (Solia S60, Biotronik, SE \& Co, KG, Berlin, Germany) delivered through a pre-shaped sheath (Selectra 3D, curve 55 or 65, Biotronik, limited market release). Ten patients (validation-group) were implanted by conventional discontinuous lead deployment. After each rotation step pacing characteristics during pacing from the inserted lead stylet (LS) were compared to connector-pin (CP) pacing. Pacing characteristics were compared to validate similar performance of both pacing methods.

Additional twenty patients were implanted according to our novel continuous pace mapping technique (feasibility-group). In this group, performance and safety of uninterrupted lead implantation during continuous pacing from the LS were investigated. The study was approved by the local ethical committee.

\subsection{Preparation of the lead and determination of the implant site.}

Preparation of the stylet-driven lead (Solia S60, Biotronik, SE \& Co, KG, Berlin, Germany) and determination of the implant site were performed as previously described.[7-9] In summary, the stylet was fully advanced to the tip of the pacing lead and maintained in this position until the end of the implant procedure. Turning the CP 10 times clockwise exposed the helix. Further torque on the inner coil was subsequently delivered by applying 10 additional clockwise rotations on the CP by using the stylet insertion tool. Finally, without loosing the built-up tension (without unwinding of the CP), the stylet insertion tool was pushed over the silicon part adjacent to the CP to maintain the build-up torque onto the outer lead body. Thereafter, the sheath with pacing lead was positioned in the right atrium. The His bundle region was delineated using the pacing lead unipolar electrograms for mapping, and its localization on fluoroscopic image was set as a reference. Next, starting from the right ventricle, the sheath and pacing lead were positioned according to the nine-partition method in the right anterior oblique view.[9] A counterclockwise rotation was performed to direct the sheath towards the RV septum. A left anterior oblique view was used to confirm a perpendicular position to the septum. From that position, and after unipolar pacing demonstrated a "W" QRS pattern in V1 with a vertical inferior lead vector and a discordant pattern in aVR and aVL, the implantation of the lead was started.

2.3 Conventional interrupted LBBAP implantation with comparison of connector-pin pacing versus lead stylet-pacing

Ten patients were implanted according to the conventional interrupted pace mapping technique (validationgroup), as previously described.[5,8] Briefly, implantation was started with 4 to 6 rapid manual clockwise rotations of the outer lead body. Subsequently, additional steps of 2 rotations were performed until successful LBBAP was demonstrated.

After the initial rotations and after each additional step, unipolar pacing characteristics were compared between pacing from the CP and from the LS. For this purpose, pacing cables were alternately connected to the CP and to the LS as a cathode, while the patient's body connection served as anode (Figure 1). Unipolar 
pacing characteristics (impedance and paced QRS morphology) and local electrograms were recorded using the Renamic programmer as generator (Biotronik, SE \& Co, KG, Berlin, Germany) and an electrophysiology recording system (BARD Labsystem, C.R Bard Inc, Lowell, MA, USA), for off-line comparison. For ethical reasons, during each rotations step pacing cables were connected to the lead stylet for unipolar LS pacing, in order to avoid lead perforation.

\subsection{Uninterrupted LBBAP implantation guided by continuous unipolar LS pacing}

Twenty patients were implanted using the novel uninterrupted pace mapping technique (feasibility-group). First, pacing cables were connected to the LS (cathode) and to the patient's body (anode) with the LS fully advanced to the tip of the lead (Figure 1B). Implantation was then performed by uninterrupted manual clockwise rotations of the outer lead body, while pacing continuously at $5 \mathrm{~V}$ output from the LS at $100 \mathrm{bpm}$ (or 10bpm over the patient's rhythm if the patient's rhythm was faster). During implantation, 12-leads ECG was recorded to monitor QRS changes (with a particular attention to V1 and V6, highlighted and magnified on the EP recording system). Rotations were continuously delivered until a right bundle branch block (RBBB) morphology was obtained in V1. Triggers and callipers were set on the EP recording system for continuous monitoring of the QRS duration (from pacing artifact to end-QRS) and pLVAT (from pacing artifact to peak R-wave in V6). Rotations were continuously delivered until right bundle branch block (RBBB) morphology was obtained in V1 with a targeted pLVAT value of $<85 \mathrm{~ms}$ (for narrow native QRS) or $<100 \mathrm{~ms}$ (for wide native QRS or a complete AV block at baseline). Beat-to-beat pacing impedance displayed on the programmer was simultaneously filmed for off-line analysis.

After continuous lead deployment, final paced QRS duration and pLVAT (measured from pacing stimulus to peak of the $\mathrm{R}$ wave in lead V6) were recorded and no further advancement of the lead was performed.

\subsection{Criteria of successful LBB capture.}

Successful LBB capture was considered in the presence of a narrow paced QRS $(<120 \mathrm{~ms})$ or in the presence of a left bundle branch potential on the sensed electrogram. When absent, short and stable pLVAT at differential high and low pacing output was used to confirm LBB capture. This was defined as pLVAT $<85 \mathrm{~ms}$ (for narrow native QRS) or $<100 \mathrm{~ms}$ (for wide native QRS or a complete AV block at baseline). [8-10]

\subsection{Data collection}

Baseline patient characteristics, ECG features, and pacing indications were collected. After implantation final pacing thresholds, R-wave sensing amplitudes, and lead impedances were measured during both uniand bipolar pacing. The paced QRS duration and pLVAT were measured. Number of implantation attempts and fluoroscopy time were recorded. All patients underwent post-procedural transthoracic echocardiographic evaluation to assess potential LBBAP implantation-related complications.

\subsection{Statistical analysis}

Categorical variables are expressed as numbers and percentages. Continuous values are reported as mean \pm 1 standard deviation. Linear regression analysis and Bland-Altman plots with assessment of systematic bias and $95 \%$ confidence intervals were used to compare LS and CP pacing characteristics. Systematic differences in measurements between the 2 modalities were assessed using Student test. All tests were two-sided and a $\mathrm{p}$ value $<0.05$ was considered as statistically significant. Statistical analyses were performed using Prism Graphpad and SPSS Statistics 24 (IBM Corporation, Armonk, NY, USA).

\section{Results}

3.1 Patients and procedural characteristics.

Patients and procedural characteristics are presented in Table 1.

3.2 Validation of LS compared to CP pacing 
In the validation-group, LBBAP was obtained in all patients with non-selective (4/10 patients, 40\%) or selective (6/10 patients, 60\%) LBB capture. Unipolar pacing impedance from the LS and CP were compared at each step of the implantation procedure and showed an excellent correlation ( $\mathrm{R}$ square $0.9481, \mathrm{p}<0.0001$ ) with an insignificant bias of $12 \pm 37 \mathrm{Ohm}$. (Figure 2) Recording of local unipolar electrograms (EGM) showed equivalent performance of LS and CP with LBB EGM recording in the same 6/10 patients (60\%) and similar morphology of the paced QRS. (Figure 3)

\subsection{Continuous lead stylet pacing to guide LBBAP}

In the feasibility-group, all patients demonstrated selective (14/20 patients, $70 \%)$ or non-selective $(6 / 20$ patients, $30 \%) \mathrm{LBB}$ capture at the end of the continuous transseptal-screwing maneuver. This was associated with a short and stable mean pLVAT of 73ms [IQR 68-80.5] and short median paced QRS duration of $120 \mathrm{~ms}$ [IQR 112-152ms]. The screwing maneuver duration was highly variable and ranged from 7.8 seconds to 27 seconds. During lead deployment the impedance evolution was recorded for off-line analysis. However, in 5 patients the recording quality was insufficient, and those impedance data were discarded.

As the lead progressed from the RV septum to the LV sub-endocardial region, a small increase followed by progressive decrease of the unipolar pacing impedance was noted in most patients (with a mean impedance difference $143 \pm 87 \mathrm{Ohm}$ ) ending with a discrete increase just before reaching final position (Figure 4). The impedance at final position ranged from 340 to $728 \mathrm{Ohm}$. Two patients showed an unexpected progressive increase in impedance from 590 to $715 \mathrm{Ohm}$ and from 405 to $674 \mathrm{Ohm}$. This was associated with a suboptimal paced QRS morphology without qR pattern in precordial ECG lead V1 in both patients, but with narrow QRS (120ms) and pLVAT (74ms) in the first patient and with clear LBB potential on local EGM and narrow QRS and PLVAT (126ms and 71ms respectively) in the second patient. No helix retraction was observed in these 2 patients.

\subsection{Performance of conventional interrupted vs. continuous implantation technique}

Excellent pacing performance was demonstrated in all patients (see table 1).

In the validation-group, conventional interrupted implantation leaded to successful LBBAP at first attempt in 7 patients (70\%). Failure of success at first attempt was caused by difficulties in further progression of the lead, after initial penetration of the helix into the RV septum. By interruption after the initial rotations, loss of torque and momentum occurred, preventing further penetration and progression of the lead through the septum.

At final position, 6/10 patients (60\%) had non-selective capture of the LBB, in the other $40 \%$ selective capture could be reached.

In the feasibility-group, continuous uninterrupted lead deployment leaded to successful LBBAP at first attempt in 18 patients (90\%), at second (1 patients, $5 \%$ ) or third attempt (1 patient, $5 \%$ ). Failure of success at first attempt was exclusively due to suboptimal orientation of the sheath, impeding a good initial penetration of the helix into the RV septum. Small corrections in the position of the sheath enabled easy implantation of the lead in patients with a first failed attempt. At final position, 6/20 patients (30\%) had non-selective capture of the LBB ( $\mathrm{p}=0.77$ vs validation-group), in the other $70 \%$, selective capture could be reached ( $\mathrm{p}=0.584$ vs validation-group). No significant differences between the validation and feasibility-groups were observed for the paced QRS duration (median QRSd 116ms [IQR109-134ms] vs median QRSd 120ms [IQR $113-144 \mathrm{~ms}$ ] respectively, $\mathrm{p}=0.4018$ ). However, the pLVAT tended to be longer in the validation compared to the feasibility-group without reaching significance (median pLVAT $87 \mathrm{~ms}$ [IQR 72-100] vs median pLVAT 73ms [IQR 69-80], respectively, $\mathrm{p}=0.0577)$. Bipolar pacing thresholds at final position were significantly lower in the feasibility-group compared to the validation-group (mean threshold $0.81 \pm 0.4 \mathrm{~V}$ vs $1.17 \pm 0.4 \mathrm{~V}$, respectively, $\mathrm{p}=0.0253$ ) with comparable median bipolar sensing $6.5 \mathrm{mV}$ [IQR 4.6-9.5] in the feasibility group vs $9.9 \mathrm{mmV}$ [IQR 6.5-14] in the validation-group, $\mathrm{p}=0.0615)$ and mean impedance values $(624 \pm 101 \mathrm{Ohm}$ in the feasibility-group vs $645 \pm 74 \mathrm{Ohm}$ in the validation-group, $\mathrm{p}=0.4339$ ). Total (skin to skin) fluoroscopic dose for device implantation was similar between groups with mean DAP $3110 \pm 1587 \mathrm{mGycm}^{2}$ in the 
validation-group, compared to $2793 \pm 4079 \mathrm{mGycm}^{2}$ in the feasibility-group ( $\left.\mathrm{p}=0.1405\right)$.

\subsection{Complications}

No perforation into the LV cavity occurred. One patient from the feasibility-group demonstrated a Dressler syndrome after implant that responded well to a conservative medical management (Echocardiography showed correct position of the LBBAP lead without perforation). No surgical repositioning was needed. All patients demonstrated stable pacing characteristics and LBB capture at discharge.

\section{Discussion}

The findings of this prospective study can be summarized as follows: First, unipolar pacing from an inserted lead stylet and from the lead connector pin demonstrated similar performance and pacing characteristics (EGM quality, pacing impedance, paced QRS morphology). Secondly, implementation of continuous unipolar stylet pacing during LBB lead implantation is feasible, safe and facilitates the transseptal positioning of stylet driven pacing leads.

Continuous unipolar pacing characteristics monitoring to guide LBBAP

Left Bundle Branch Area Pacing (LBBAP) involves deep transeptal screwing of the lead until the LV subendocardium is reached. The exact number of rapid rotations of the lead during this manoeuvre remains highly variable and depends among other factors of the septal wall thickness, the penetration angle of the lead in the septum, and the progression speed of the lead through the septum. Regular control of the paced QRS morphology and unipolar pacing impedance is key to avoid perforation in the LV cavity and confirm engagement of the left septal conduction system. Typically, as the pacing lead advance into the septum, the "W" shaped QRS morphology in lead V1 gradually changes towards an incomplete right bundle branch block morphology ( $\mathrm{qR} / \mathrm{rsR}$ ') in lead V1. This indicates appropriate progression of the lead through the septum. Simultaneously, the global QRS duration and the initial QRS slurring or pLVAT in lead V6 decrease as the LBB area is approached. Unless a transseptal perforation has occurred, the unipolar lead impedance progressively rises and falls (slightly) before reaching the LV sub-endothelium. The intermittent pace mapping strategy impose the rapid rotations of the outer lead body to be repeatedly interrupted to connect the programmer (via crocodile clips) to the connector pin for pacing. If no LV cavity perforation has occurred and if criteria for LBBAP are not met after a first initial lead engagement, the crocodile clips are disconnected, and the lead can be carefully further advanced until next evaluation. With the loss of momentum, the repetition of these steps may compromise distal torque transmission. This is particularly true with stylet-driven leads, which are non-isodiametric and more susceptible to accumulate torsional forces. As a consequence, further lead progression might be compromised, and late retraction of the helix can occur with eventual loss of LBB capture or inappropriate fixation. Finally, the repetition of the pace mapping after each set of lead rotations prolongs the procedure and increases the risk of sheath dislodgement. Therefore continuous monitoring of unipolar pacing characteristics without interruption of screwing-in of the lead is highly desirable.

Pace mapping options with the lumen-less pacing lead

In the early development of LBBAP, Jastrzebski and others, using the lumen-less Medtronic 3830 lead, proposed to continuously pace mapping during LBB implant by keeping the crocodile connector onto the lead CP.[10-12] Unfortunately, given the lack of commercially available revolving connectors for the distal pin, practical application of this technique was limited by the unstable connection and more importantly by the significant risk of fracture between the distal pin and the inner conducting wire. Accordingly, further practical development focused on the recognition of "irritative" PVCs generated during the transseptal screwing of the LBB lead. Ponnusamy et al. proposed monitoring of the change in PVC during lead positioning (dubbed by the authors as "template beats"). In their study, only two-thirds of the patients demonstrated PVCs during rapid lead deployment. Moreover, their occurrence was inconstant and unpredictable.[5,13] Similarly, Jastrzebski et al. proposed instead of interrupted pace mapping after each set of lead rotations, to continue the initial lead rotations until typical relatively narrow RBB PVCs (called "fixation beats") appears.[11] 
Although very specific, "fixation beats" appear only when the lead has already reached the LBB area and indicates thus that lead rotations should be immediately stopped to prevent LV perforation. As recognized by de authors, this technique can be challenging (especially in RBBB patients) and needs a strict monitoring of their occurrence to avoid perforation.

Pace mapping options with stylet driven pacing leads

In contrast, using stylet-driven leads, the novel approach we are proposing offers the possibility of continuous monitoring of unipolar pacing characteristics in all patients at a predetermined rate, allowing thus for an improved control during the transseptal positioning of the lead. As demonstrated in the present study, we found that pacing from the inserted lead stylet is feasible and reliable and is associated with a very low risk of implant failure due to inadequate progression of the lead or sheath dislodgement. The safety of this approach is further demonstrated by the absence of perforation into the LV cavity. As show in our study continuous LS pacing during implantation allows for real-time monitoring of the transition of QRS morphology towards a RBBB morphology, guided by the pLVAT value. (Figure 5)

Moreover, continuous monitoring of the pacing impedance during lead implantation might allow for the early detection of inadvertent helix-retraction, which would be associated with a significant increase in impedance during the transseptal screwing.

Ideal pacing lead for LBBAP

Comparison of both stylet-driven and lumen-less pacing leads, has demonstrated similar performance. Yet, the lack of a revolving connector pin represents in our opinion a limitation with the current implant technique using lumen-less pacing leads. Beyond some advantages in terms of steerability and septum penetration, and despite the fragility of the screwing mechanism[14], we have found the possibility of continuous pacemapping during transseptal implantation a major advantage when using stylet driven pacing leads. In our opinion, future development of specific leads for LBBAP should allow for continuously monitoring of the pacing characteristics.

Limitations

The present study has several limitations that should be acknowledged. First, we tested only the Biotronik Solia S60 pacing lead in combination with the regular stylet delivered in the same packaging. Our results can therefore not be transposed to other stylet-driven leads or stylet and lead combination. Secondly, for ethical reasons, even in the validation-group, LS pacing was used in between the pace-mapping steps to inform the implanter of the progression of the lead. Therefore, we cannot consider that the LS pacing strategy was strictly compared to the traditional interrupted pace-mapping approach. Finally, our study is underpowered to demonstrate the superiority of one of the two techniques in terms of complications.

\section{Conclusion}

Unipolar pacing from the inserted lead stylet allows for accurate determination of pacing impedance and generates similar paced QRS morphologies and sensed electrograms, compared to pacing form the connector pin. Continuous unipolar lead stylet pacing allows for real-time monitoring of impedance and paced QRS morphology, which facilitates a safe and successful LBBAP lead implantation.

\section{References}

1. Huang W, Su L, Wu S, Xu L, Xiao F, Zhou X, et al. A Novel Pacing Strategy With Low and Stable Output: Pacing the Left Bundle Branch Immediately Beyond the Conduction Block. Can J Cardiol. Canadian Cardiovascular Society; 2017;33: 1736.e1-1736.e3. doi:10.1016/j.cjca.2017.09.013

2. Vijayaraman P, Subzposh FA, Naperkowski A, Panikkath R, John K, Mascarenhas V, et al. Prospective evaluation of feasibility and electrophysiologic and echocardiographic characteristics of left bundle branch area pacing. Hear Rhythm. Elsevier Inc.; 2019;16: 1774-1782. doi:10.1016/j.hrthm.2019.05.011 
3. Li Y, Chen K, Dai Y, Li C, Sun Q, Chen R, et al. Left bundle branch pacing for symptomatic bradycardia: Implant success rate, safety, and pacing characteristics. Hear Rhythm. Heart Rhythm Society; 2019;16: 1758-1765. doi:10.1016/j.hrthm.2019.05.014

4. Li X, Li H, Ma W, Ning X, Liang E, Pang K, et al. Permanent left bundle branch area pacing for atrioventricular block: Feasibility, safety, and acute effect. Hear Rhythm. Heart Rhythm Society; 2019;16: 1766-1773. doi:10.1016/j.hrthm.2019.04.043

5. Ponnusamy SS, Arora V, Namboodiri N, Kumar V, Kapoor A, Vijayaraman P. Left bundle branch pacing: A comprehensive review. J Cardiovasc Electrophysiol. 2020;31: 2462-2473. doi:10.1111/jce.14681

6. Vijayaraman P, Ponnusamy SS, Cano Ó, Sharma PS, Naperkowski A, Subsposh FA, et al. Left Bundle Branch Area Pacing for Cardiac Resynchronization Therapy: Results From the International LBBAP Collaborative Study Group. JACC Clin Electrophysiol. 2021;7: 135-147. doi:10.1016/j.jacep.2020.08.015

7. Pooter J De, Calle S, Timmermans F. Left bundle branch area pacing using stylet-driven pacing leads as compared to lumen-less leads. 2020; $1-11$.

8. Huang W, Chen X, Su L, Wu S, Xia X, Vijayaraman P. A beginner's guide to permanent left bundle branch pacing. Hear Rhythm. Elsevier Inc.; 2019;16: 1791-1796. doi:10.1016/j.hrthm.2019.06.016

9. Zhang J, Wang Z, Zu L, Cheng L, Su R, Wang X, et al. Simplifying Physiological Left Bundle Branch Area Pacing Using a New Nine-Partition Method. Can J Cardiol. Canadian Cardiovascular Society; 2020; doi:10.1016/j.cjca.2020.05.011

10. Jastrzebski M, Kiełbasa G, Curila K, Moskal P, Bednarek A, Rajzer M, et al. PhysiologyBased Electrocardiographic Criteria for Left Bundle Branch Capture. Hear Rhythm. 2020; doi:10.1101/2020.12.24.20248827

11. Jastrzebski M, Kiełbasa G, Moskal P, Bednarek A, Kusiak A, Sondej T, et al. Fixation beats - a novel marker for reaching the left bundle branch area during deep septal lead implantation. Hear Rhythm. 2020; doi:10.1101/2020.10.04.20206573

12. Jastrzebski M, Moskal P. Reaching the left bundle branch pacing area within 36 heartbeats. Kardiol Pol. 2021;79: 587-588. doi:10.33963/KP.15914

13. Ponnusamy SS, Vijayaraman P. Left bundle branch pacing guided by premature ventricular complexes during implant. Hear Case Reports. Elsevier Inc.; 2020;6: 850-853. doi:10.1016/j.hrcr.2020.08.010

14. le Polain de Waroux JB, Wielandts JY, Gillis K, Hilfiker G, Sorgente A, Capulzini L, et al. Repositioning and extraction of stylet-driven pacing leads with extendable helix used for left bundle branch area pacing. J Cardiovasc Electrophysiol. 2021;32: 1464-1466. doi:10.1111/jce.15030

Table and figure legends

Table 1. Patients and procedural characteristics. ${ }^{*}$ Pulse-width $=0.4 \mathrm{~ms}$.

AVB AV block; BMI body mass index; DAP dose area product; IVSd interventricular septum in diastole; LADplax left atrial diameter in parasternal long axis view; LBB left bundle branch; LBBB left bundle branch block; LVEF left ventricular ejection fraction; pLVAT peak left ventricular activation time; pQRSd paced QRS duration; RBBB right bundle branch block.

Figure 1. Illustration of pacing cables connected to the connector pin (cathode) and to the patient's body (anode) for conventional interrupted implantation (A) versus pacing cables connected to the lead stylet (cathode) with the stylet fully advanced to the tip of the lead and connected to the patient's body (anode) for continuous implantation (B).

Figure 2. Linear regression and limits of agreement between estimates of unipolar pacing impedance on connector pin vs lead stylet. $\mathrm{CP}=$ connector pin. 
Figure 3. Connector pin and lead stylet derived unipolar his-electrograms (his-EGM), left bundle branch electrograms (LBB-EGM) and QRS morphology during pacing at final LBBAP position with nonselective (NS) and selective (S) LBBAP capture.

Figure 4. Evolution of beat-to-beat unipolar lead stylet pacing impedance values $(\Omega)$ during continuous uninterrupted implantation of the LBBAP lead. Each line represents an individual patient from the feasibility-group. LS: lead stylet.

Figure 5. Unedited continuous tracing demonstrating LS paced QRS morphology during continuous LBBAP lead implantation. Selected values of the beat-to-beat monitored unipolar pacing impedance $(\Omega)$ and pLVAT (ms) are displayed above and under the tracing. ECG speed 13mm/sec. LS: lead stylet. pLVAT: peak left ventricular activation time.

Table 1. Patient characteristics

\begin{tabular}{|c|c|c|c|}
\hline & Validation Group $(\mathrm{n}=10)$ & $\begin{array}{l}\text { Feasibility } \\
\text { Group }(\mathrm{n}=20)\end{array}$ & p-value \\
\hline $\begin{array}{l}\text { Age (years), mean } \pm \\
\text { SD }\end{array}$ & $78 \pm 9$ & $74 \pm 16$ & 0.6592 \\
\hline Female gender, $\mathrm{n}(\%)$ & $5(50)$ & $8(40)$ & 0.602 \\
\hline $\begin{array}{l}\mathrm{BMI}\left(\mathrm{kg} / \mathrm{m}^{2}\right), \text { mean } \pm \\
\mathrm{SD}\end{array}$ & $27 \pm 5$ & $28 \pm 4$ & 0.7084 \\
\hline \multicolumn{4}{|l|}{ Pacing indication } \\
\hline Bradycardia, n(\%) & $8(80)$ & $17(85)$ & 0.729 \\
\hline AVB II / III, n(\%) & $2(20) / 3(30)$ & $2(10) / 8(40)$ & 0.536 \\
\hline Heartfailure, $\mathrm{n}(\%)$ & $2(20)$ & $6(30)$ & 0.559 \\
\hline \multicolumn{4}{|l|}{ ECG characteristics } \\
\hline Sinus rhythm, n(\%) & $6(60)$ & $12(60)$ & 1.000 \\
\hline Atrial fibrillation, $\mathrm{n}(\%)$ & $4(40)$ & $8(40)$ & 1.000 \\
\hline $\begin{array}{l}\text { PR interval (ms), } \\
\text { median[IQR] }\end{array}$ & $198[177-360]$ & $226[175-246]$ & 0.6236 \\
\hline $\begin{array}{l}\text { QRSd }(\mathrm{ms}), \text { mean } \pm \\
\mathrm{SD}\end{array}$ & $126 \pm 38$ & $134 \pm 41$ & 0.6440 \\
\hline LBBB, $n(\%)$ & $1(10)$ & $5(25)$ & 0.393 \\
\hline $\mathrm{RBBB}, \mathrm{n}(\%)$ & $2(20)$ & $6(30)$ & 0.559 \\
\hline \multicolumn{4}{|l|}{$\begin{array}{l}\text { Echocardiographic } \\
\text { parameters }\end{array}$} \\
\hline $\operatorname{LVEF}(\%)$, mean $\pm \mathrm{SD}$ & $52 \pm 13$ & $54 \pm 15$ & 0.8079 \\
\hline $\begin{array}{l}\text { LADplax }(\mathrm{mm}), \text { mean } \\
\pm \mathrm{SD}\end{array}$ & $45 \pm 5$ & $44 \pm 7$ & 0.9186 \\
\hline $\begin{array}{l}\text { IVSd }(\mathrm{mm}), \text { mean } \pm \\
\mathrm{SD}\end{array}$ & $11 \pm 2$ & $11 \pm 2$ & 0.7060 \\
\hline \multicolumn{4}{|l|}{$\begin{array}{l}\text { Procedural } \\
\text { characteristics }\end{array}$} \\
\hline $\begin{array}{l}\text { Successful } \\
\text { implantation, n(\%) }\end{array}$ & $10(100)$ & $20(100)$ & \\
\hline $\begin{array}{l}\text { Implantation attempts, } \\
\text { mean } \pm \text { SD }\end{array}$ & $2 \pm 1$ & $1 \pm 0$ & 0.1448 \\
\hline $\begin{array}{l}\text { Procedure time }(\min ), \\
\text { mean } \pm \mathrm{SD}\end{array}$ & $87 \pm 39$ & $68 \pm 29$ & 0.2334 \\
\hline $\begin{array}{l}\text { Fluoroscopy time }(\mathrm{sec}) \text {, } \\
\text { median[IQR] }\end{array}$ & $8[3-17]$ & $4[2-7]$ & 0.2050 \\
\hline $\operatorname{DAP}\left(\mathrm{mGycm}^{2}\right)$ & $3110 \pm 1587$ & $2793 \pm 4078$ & 0.1405 \\
\hline
\end{tabular}




\begin{tabular}{|c|c|c|c|}
\hline & Validation Group $(\mathrm{n}=10)$ & $\begin{array}{l}\text { Feasibility } \\
\text { Group }(n=20)\end{array}$ & $\mathrm{p}$-value \\
\hline \multicolumn{4}{|l|}{ Final parameters } \\
\hline $\begin{array}{l}\text { Implant depth }(\mathrm{mm}) \text {, } \\
\text { median[IQR] }\end{array}$ & $14.4[11.8-14.8]$ & 15 [11.8-15] & 0.1788 \\
\hline Bipolar Pacing & $1.2 \pm 0.4$ & $0.8 \pm 0.4$ & 0.0253 \\
\hline $\begin{array}{l}\text { Threshold }(\mathrm{V})^{*} \\
\text { Sensing }(\mathrm{mV}) \text {, } \\
\text { median[IQR] }\end{array}$ & $10[7-14]$ & $7[5-10]$ & 0.0615 \\
\hline $\begin{array}{l}\text { Impedance }(\mathrm{Ohm}) \\
\text { mean } \pm \mathrm{SD}\end{array}$ & $645 \pm 74$ & $624 \pm 101$ & 0.4339 \\
\hline LBB potential, $\mathrm{n}(\%)$ & $6(60)$ & $7(35)$ & 0.193 \\
\hline $\begin{array}{l}\text { pQRSd (ms) } \\
\text { median[IQR] }\end{array}$ & $116[110-136]$ & $1120[113-144]$ & 0.4018 \\
\hline $\begin{array}{l}\text { pLVAT (ms), } \\
\text { median[IQR] }\end{array}$ & $87[72-100]$ & $73[69-80]$ & 0.0577 \\
\hline LBB capture & & & \\
\hline Non-selective, $\mathrm{n}(\%)$ & $4(40)$ & $6(30)$ & 0.770 \\
\hline Selective, $\mathrm{n}(\%)$ & $6(60)$ & $14(70)$ & 0.584 \\
\hline
\end{tabular}

\section{Figure 1.}
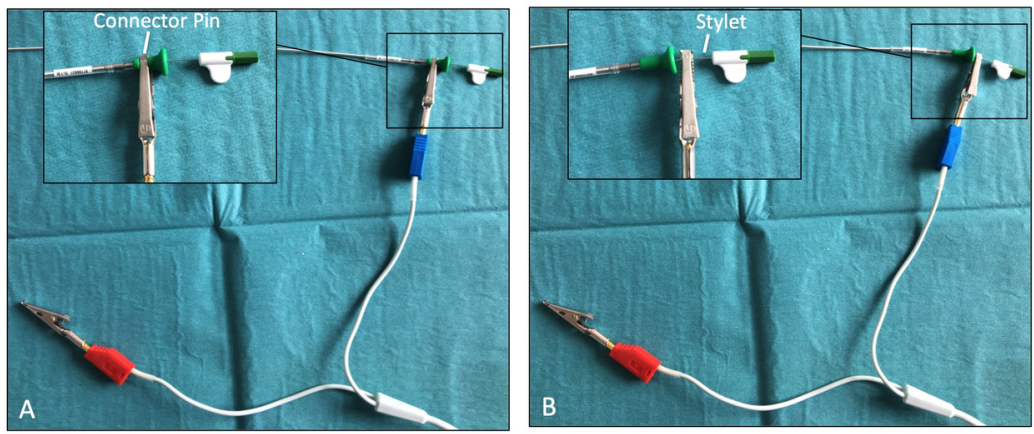

\section{Figure 2.}


Correlation unipolar pacing impedance Connector Pin vs Lead Stylet

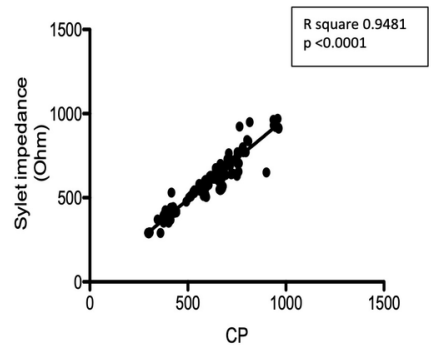

Bland-Altman unipolar pacing impedance Connector Pin vs Lead Stylet

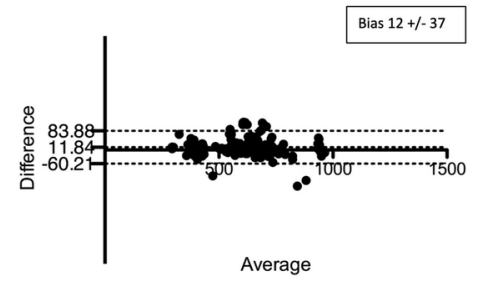

Figure 3.
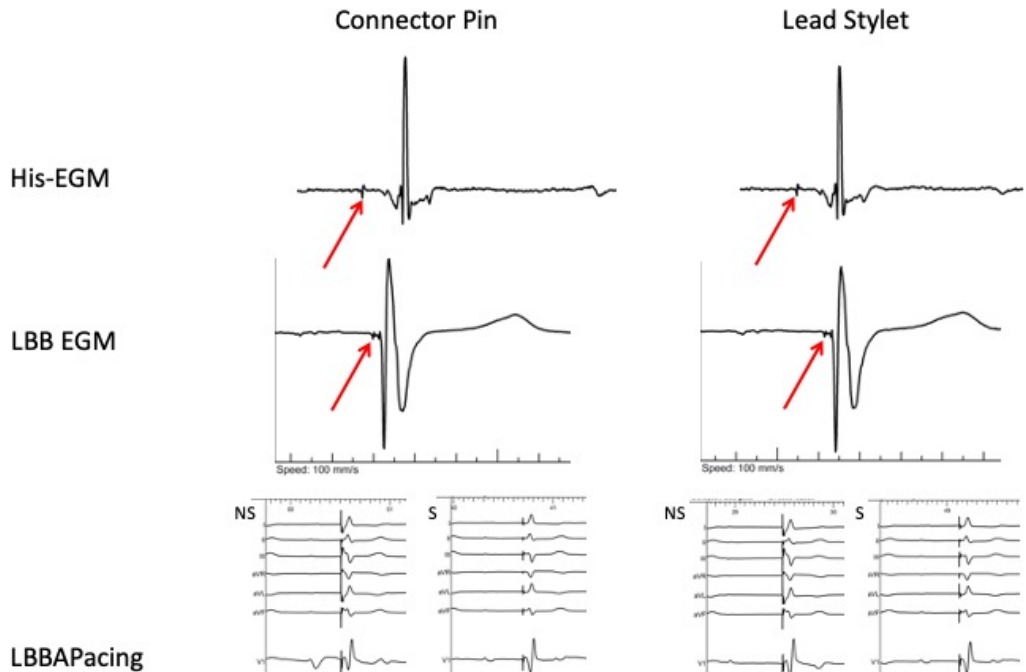

NS

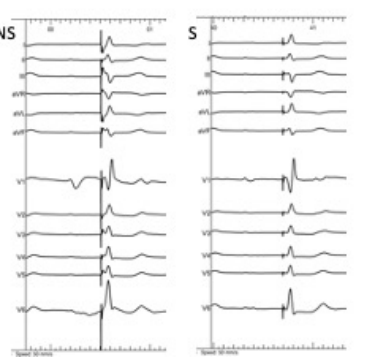

NS

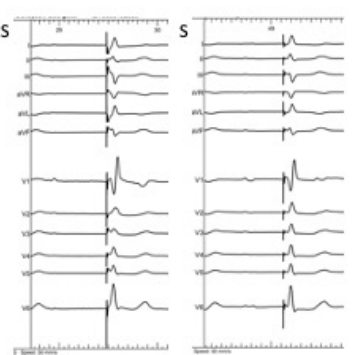

Figure 4. 


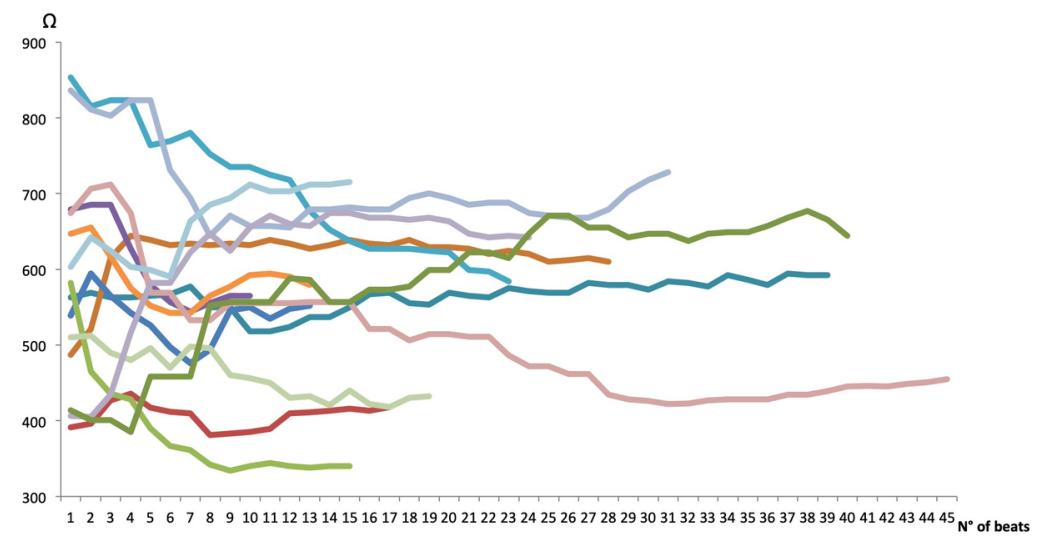

Figure 5.

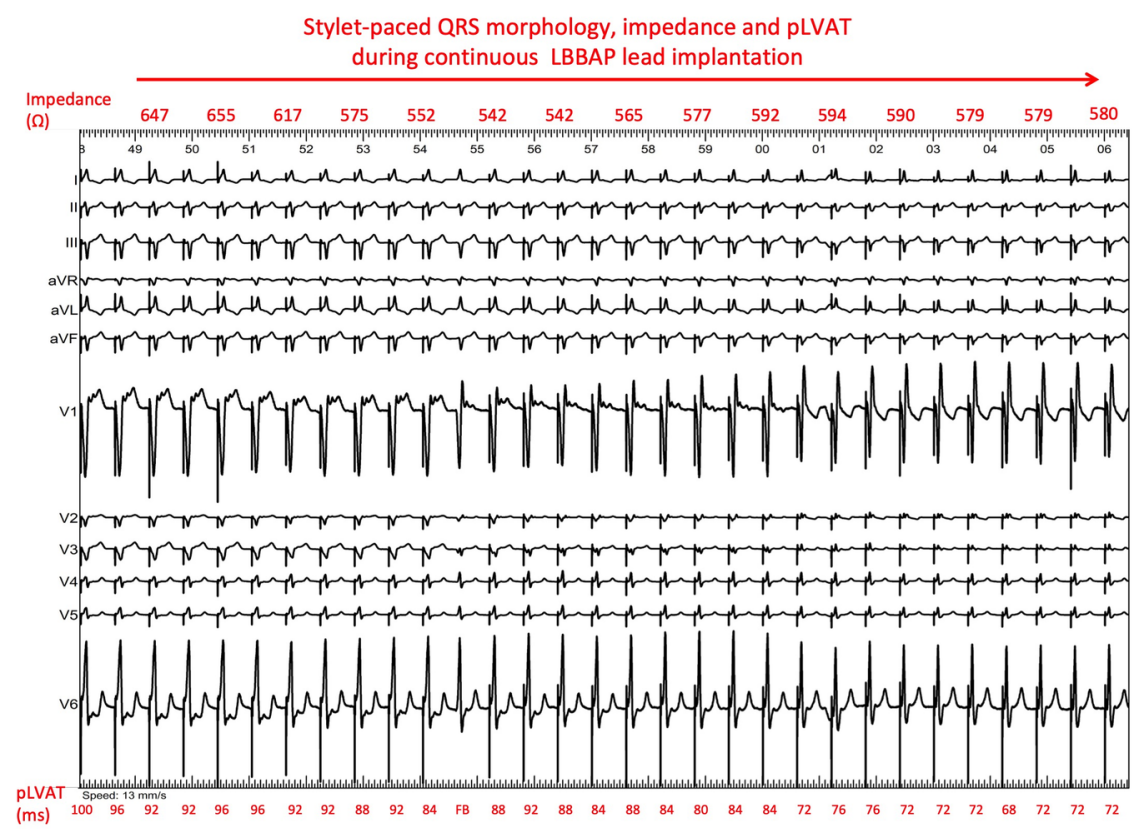

